\title{
Design of Novel Metal Nanostructures for Broadband Solar Energy Conversion
}

\author{
Kristine A. Zhang, David Ma, Ying-Chih Pu, and Yat Li \\ Department of Chemistry and Biochemistry, University of California, Santa Cruz, CA 95064, USA \\ Correspondence should be addressed to Yat Li; yatli@ucsc.edu
}

Received 13 September 2014; Accepted 29 December 2014

Academic Editor: Guillermo Moyna

Copyright ( 2015 Kristine A. Zhang et al. This is an open access article distributed under the Creative Commons Attribution License, which permits unrestricted use, distribution, and reproduction in any medium, provided the original work is properly cited.

\begin{abstract}
Solar power holds great potential as an alternative energy source, but current photovoltaic cells have much room for improvement in cost and efficiency. Our objective was to develop metal nanostructures whose surface plasmon resonance (SPR) spectra closely match the solar spectrum to enhance light absorption and scattering. We employed the finite-difference time-domain simulation method to evaluate the effect of varying key parameters. A novel nanostructure with SPR absorption matching a region of the solar spectrum (300 to $1500 \mathrm{~nm}$ ) that contains $90 \%$ of solar energy was successfully designed. This structure consists of a large gold-silica core-shell structure with smaller gold nanoparticles and nanorods on its surface. Such complex nanostructures are promising for broad and tunable absorption spectra. In addition, we investigated the SPR of silver nanoparticle arrays, which can achieve scattering close to the solar spectrum. We demonstrated an improvement in efficiency of over $30 \%$ with optimal nanoparticle radius and periods of $75 \mathrm{~nm}$ and $325 \mathrm{~nm}$, respectively. In combination, our studies enable high-efficiency, tunable, and cost-effective enhancement of both light absorption and scattering, which has potential applications in solar energy conversion as well as biomedical imaging.
\end{abstract}

\section{Introduction}

As the human population grows and industrialization expands, the demand for energy is increasing at an unprecedented pace while fossil fuel, the primary and nonrenewable source of energy, is being depleted at an increasing rate. Furthermore, $\mathrm{CO}_{2}$ emissions due to fossil fuel consumption have detrimental consequences for the environment and the economy.

The search for alternative and ideally renewable energy is therefore critical and currently at the forefront of science and technology. Among the different alternative energy options, solar energy is arguably the most attractive. The energy supplied by the Sun is practically unlimited and widely available and has no harmful environmental effects [1]. In addition, solar energy can potentially be produced with improved efficiency and at low costs, through large-scale manufacturing and use of inexpensive materials [1]. It can also be used to generate chemical fuels that can be stored via electrolysis or photoelectrochemical (PEC) processes [2].
Crystalline silicon-based solar cells, which currently make up the majority of the market, have a decent light to electricity conversion efficiency of about $10-20 \%$ [3]. However, the high-purity silicon needed for high-efficiency cells is expensive [4], and this issue is amplified by the bulk quantities of silicon necessary for the production of crystalline silicon-based solar cells. To address this issue, alternative materials are necessary for long-term sustainable energy solutions.

We focused primarily on two classes of materials that have shown promise for low-cost and efficient energy conversion: metal oxides and amorphous (thin-film) silicon.

Metal oxides (MOs) have the major advantages of low cost and high stability. However, most MOs do not absorb visible light due to their large electronic bandgap [5]. Several approaches have been developed to address this issue, for example, using molecules like dyes or semiconductor quantum dots (QDs) as sensitizers to help absorb visible light and to transfer the electrons generated to the MOs $[4,6]$. While the solar conversion efficiency has reached $11 \%$ with 
dye-sensitized and about 5\% for QD-sensitized solar cells in research laboratories $[7,8]$, dyes and QDs tend to be unstable over long periods of exposure to heat or light $[4,6,9]$.

Another class of solar cells that has shown promise for reduced-cost energy conversion is thin-film, or amorphous, silicon solar cells. Currently, over $80 \%$ of solar cells produced are crystalline silicon solar cells [4], with the main advantages of relatively high-efficiency, stability, and reliability. Nevertheless, their usage is limited by their high cost. The use of amorphous or thin-film silicon solar cells reduces the need for bulk quantities of expensive, highly purified silicon but results in a decrease in energy conversion efficiency as well because light is not completely absorbed during a single passage through the solar cell [10].

Recently, metal nanostructures (MNs) with high thermal and photo stability have been considered as potential sensitizers for MO and as enhancers for amorphous silicon solar cells [11-15]. While the underlying mechanisms are not yet well understood, the observed photoactivity in MO solar cells appears to be convincing and encouraging, and enhanced silicon solar cells have demonstrated noticeable increases in light absorption as well $[6,10,11,16]$. At present, there are several proposed models for the photocurrent observed from $\mathrm{MN}$-sensitized or MN-enhanced solar cells, including hot electron injection [11, 16], local field enhancement [17], and enhanced light absorption of bandgap states in the MO due to enhanced light scattering by the MNs [6].

Regardless of which mechanism is truly responsible for the observed photocurrent or enhancement, light absorption and scattering of the MNs are sure to play an important role. For the purposes of solar energy conversion, it is crucial that the absorption and scattering spectra of the MNs match the solar emission spectrum for optimal photocurrent generation or enhancement. Specifically, it has been suggested that sensitization of MO solar cells depends on absorption, while enhancement of silicon solar cells is dependent on light scattering.

The absorption and scattering of light by MNs in the visible and near-IR regions are primarily determined by surface plasmon resonance (SPR), which is the collective oscillation of conduction band electrons in response to an electromagnetic field, such as light. As a result, the SPR of MNs is strongly dependent on the chemical nature of the metal, its shape, and, to a lesser degree, its size. One can take advantage of the strong shape dependence of SPR to design MNs with desired SPR spectra [18-20].

Most of the MNs studied to date exhibit light absorption that is far narrower than the solar spectrum. Designing and synthesizing MNs with absorption or scattering to match the solar spectrum is highly desirable in order to improve solar conversion efficiency. Although it has been demonstrated that arrays of silver nanoparticles can have SPR scattering close to the solar spectrum, no MNs have been designed with SPR absorption matching the solar spectrum, to the best of our knowledge. Due to the complex shape and size dependence of SPR absorption, it is challenging to predict what the best structure should be for such a purpose.
In this project, we applied the finite-difference timedomain (FDTD) computational method to study the SPR properties of a variety of different NS and to test their reliance on a number of variables, such as shape, size, and material. For MO solar cells, we took the first step towards developing MNs with SPR absorption that matches the solar spectrum as closely as possible by designing a novel complex MN with SPR that exhibits an excellent match to the solar spectrum. For amorphous silicon solar cells, we evaluated several factors to optimize the SPR properties of Ag NP arrays for greater efficiency enhancement. In addition, we developed a new strategy to design cost-efficient arrays by limiting the amount of material necessary for NSs while retaining their enhancement. Our work demonstrates the potential of $\mathrm{MN}$ sensitized metal oxide and MN-enhanced amorphous silicon solar cells for cost-effective and high-efficiency solar energy conversion.

\section{Computational Method}

When the frequency of light on a metal nanostructure matches the natural frequency of conduction band electrons oscillating against the restoring force of positive nuclei in the metal, light absorption and scattering occur, known as SPR. The resonant wavelength and magnitude of light absorption and scattering depend on the shape, size, and material of a nanostructure, as well as the dielectric property of the surrounding medium [21]. Analytical solutions of Maxwell's equations that describe SPR exist only for simple shapes like the sphere and infinitely long cylinder [22]. To study the SPR of complex MNs requires numerical solution of Maxwell's equations with the proper boundary conditions.

The FDTD method has been extensively applied for studying light-matter interaction to simulate the absorption and scattering cross sections as well as the electromagnetic (EM) field distribution in the vicinity of the nanostructure [23-26]. It solves Ampere's current law and MaxwellFaraday's equation in differential form, in a region with no charge and no current, as shown in

$$
\frac{\partial E}{\partial t}=\frac{1}{\varepsilon} \nabla \times H \quad \frac{\partial H}{\partial t}=-\frac{1}{\mu} \nabla \times E,
$$

where $E$ and $H$ are the electrical and magnetic fields and $\varepsilon$ and $\mu$ are the permittivity and permeability of the dielectric medium.

The FDTD is implemented by dividing space into grids known as Yee cells [27] and quantizing time into small steps, where each step represents the time required for the EM field to travel from one Yee cell to the next. Illustrating the FDTD algorithm using a one-dimensional example, (1) can be reduced into

$$
\frac{\partial E_{x}}{\partial t}=-\frac{1}{\varepsilon} \frac{\partial H_{y}}{\partial z} \quad \frac{\partial H_{y}}{\partial t}=-\frac{1}{\mu} \frac{\partial E_{x}}{\partial z} .
$$




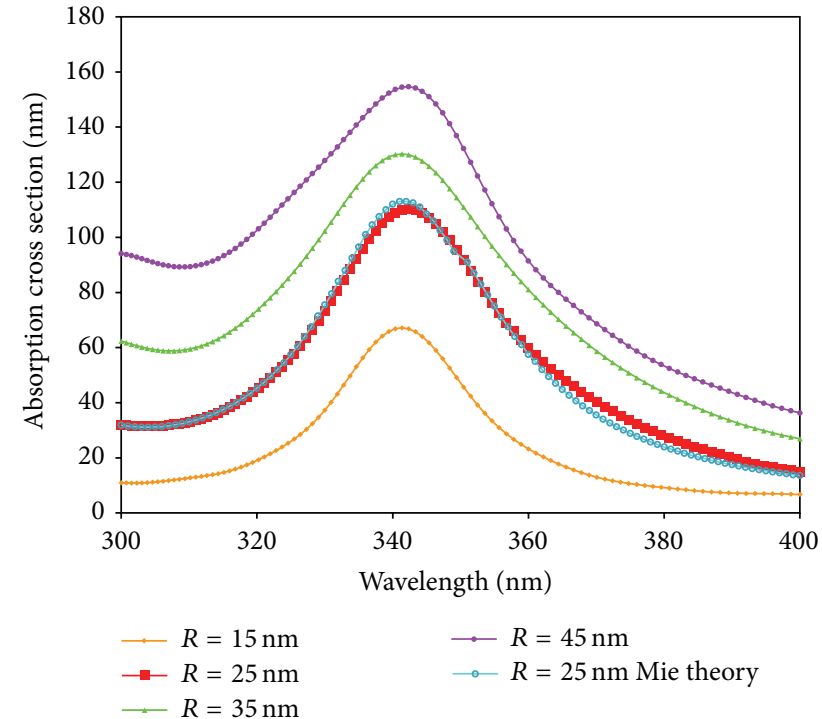

FIGURE 1: Silver nanowire: absorption as a function of radius from $300 \mathrm{~nm}$ to $400 \mathrm{~nm}$.

Discretizing the space and time partial derivatives in (2) using the central difference approximation and rearranging the terms, we get [28]

$$
\begin{aligned}
& \left.E_{x}\right|_{k} ^{n+1}=\left.E_{x}\right|_{k} ^{n}-\frac{\Delta t}{\varepsilon}\left(\frac{\left.H_{y}\right|_{k+1 / 2} ^{n+1 / 2}-\left.H_{y}\right|_{k-1 / 2} ^{n+1 / 2}}{\Delta z}\right), \\
& \left.H_{y}\right|_{k+1 / 2} ^{n+1 / 2}=\left.H_{y}\right|_{k+1 / 2} ^{n-1 / 2}-\frac{\Delta t}{\mu}\left(\frac{\left.E_{x}\right|_{k+1} ^{n}-\left.E_{x}\right|_{k} ^{n}}{\Delta z}\right),
\end{aligned}
$$

where $\left.E_{x}\right|_{k} ^{n}$ is the $x$ component of the $E$ field at space-grid $k$ and time step $n$ and $\left.H_{y}\right|_{k+1 / 2} ^{n+1 / 2}$ is the $y$ component of the $H$ field at space-grid $(k+1 / 2)$ and the time step $\left.(k+1 / 2) \cdot E_{x}\right|_{k} ^{n}$ and $\left.H_{y}\right|_{k+1 / 2} ^{n+1 / 2}$ are shifted in space by half a cell and in time by half a time step to form a central difference approximation of the partial derivative in order to achieve the second order accuracy $[27,28]$. Equations (3a) and (3b), expanded to two or three dimensions, are the basis for FDTD simulation that solves for EM fields at discrete points in space and steps in time following a leapfrog scheme.

Our simulations employ a tool named as FDTD Solutions, developed by Lumerical, to compute the absorption and scattering spectra of nanoparticles (NPs, assumed spherical) which relate the absorbed and scattered power to that of the incident light [29]:

$$
\begin{gathered}
\sigma_{\mathrm{abs}}=\frac{P_{\mathrm{abs}}}{I_{\mathrm{inc}}}, \quad \sigma_{\text {scat }}=\frac{P_{\mathrm{scat}}}{I_{\mathrm{inc}}}, \\
\sigma_{\mathrm{ext}}=\sigma_{\mathrm{abs}}+\sigma_{\text {scat }},
\end{gathered}
$$

where $P_{\text {abs }}, P_{\text {scat }}$, and $I_{\text {inc }}$ are the power absorbed, power scattered, and incident density, respectively. The extinction cross section is the sum of the absorption and scattering.

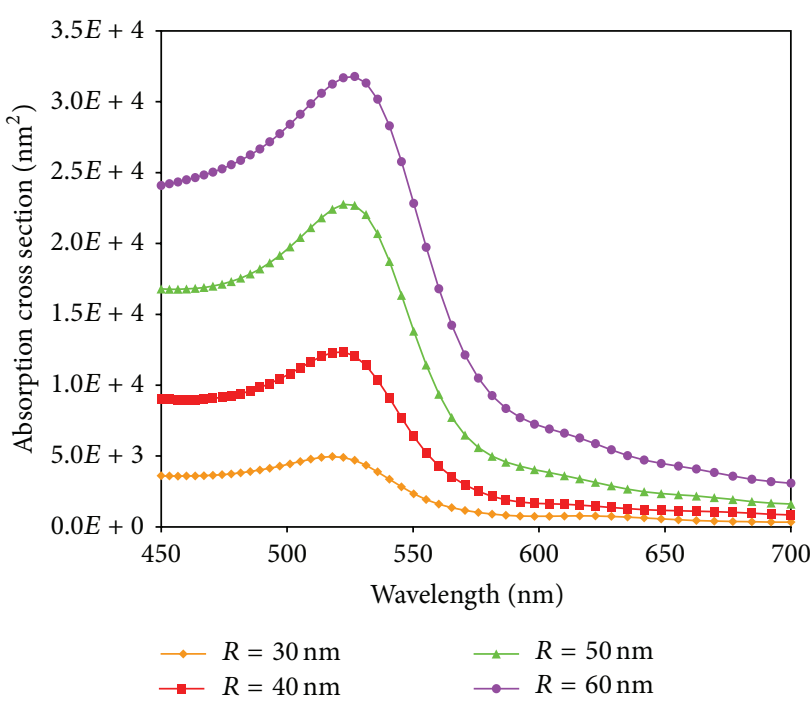

FiguRE 2: Gold sphere: absorption as a function of radius from 450 to $700 \mathrm{~nm}$.

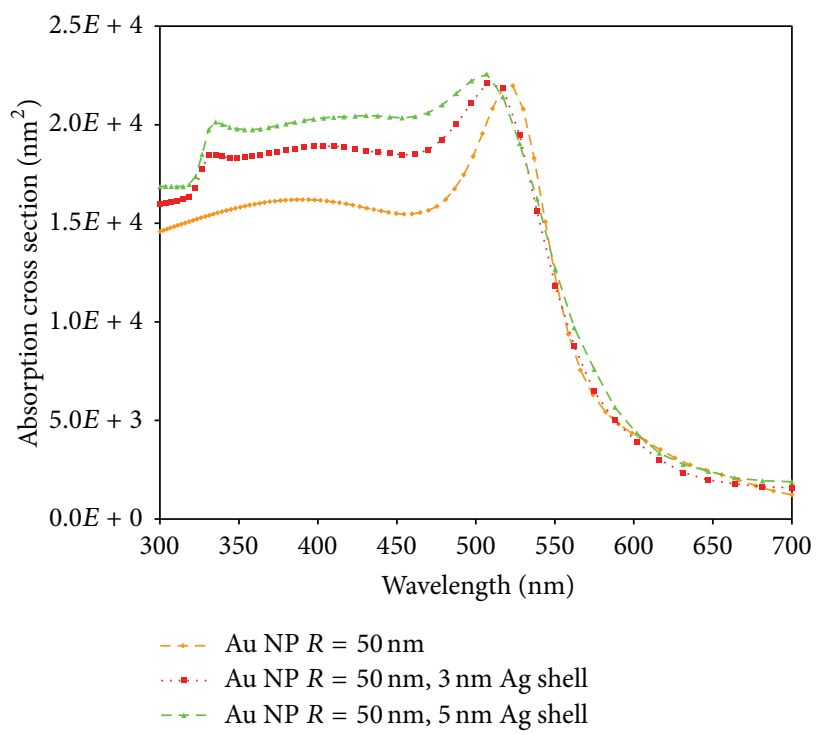

FIgure 3: Comparison of SPR absorption between Au NPs with constant radius $50 \mathrm{~nm}$ and no shell or with Ag shells of varying thickness, from $300 \mathrm{~nm}$ to $700 \mathrm{~nm}$.

Unless it is specified otherwise, our simulations apply Total-Field Scattered-Field (TFSF) source propagated in the $Y$ direction. The space and time step sizes are carefully controlled to satisfy the space and time stability conditions $[30,31]$ :

$$
\text { Space step: } \Delta_{\max } \leq \frac{\lambda}{10} \text {, }
$$

$$
\text { Time step: } \Delta t \leq \frac{1}{c \sqrt{1 /\left(\Delta_{x}^{2}+\Delta_{y}^{2}+\Delta_{z}^{2}\right)}},
$$




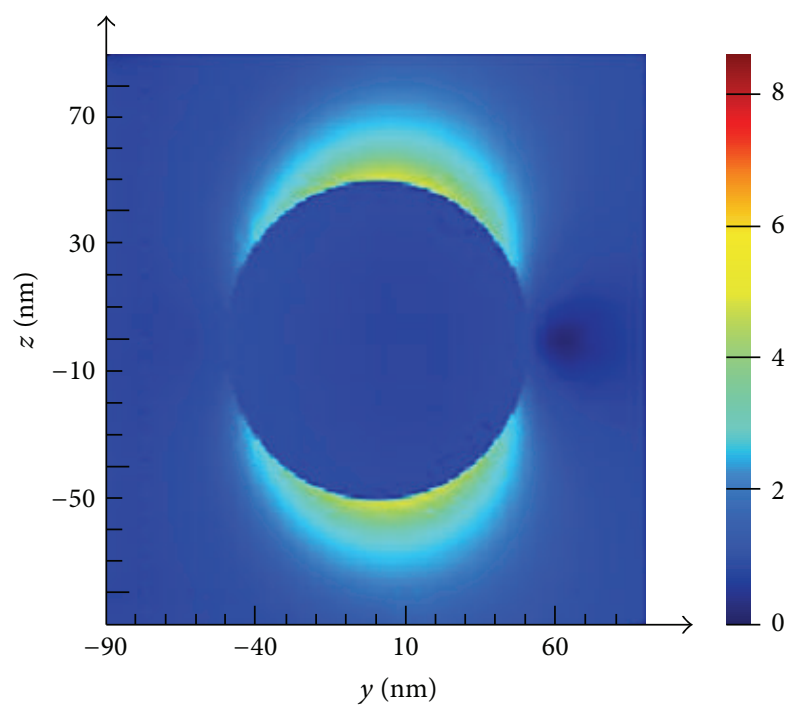

(a)

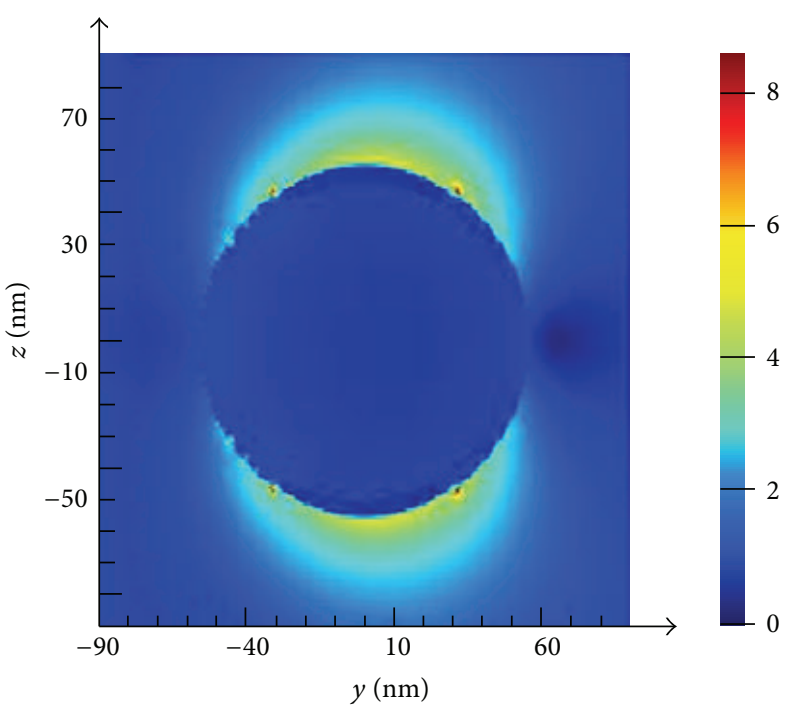

(b)

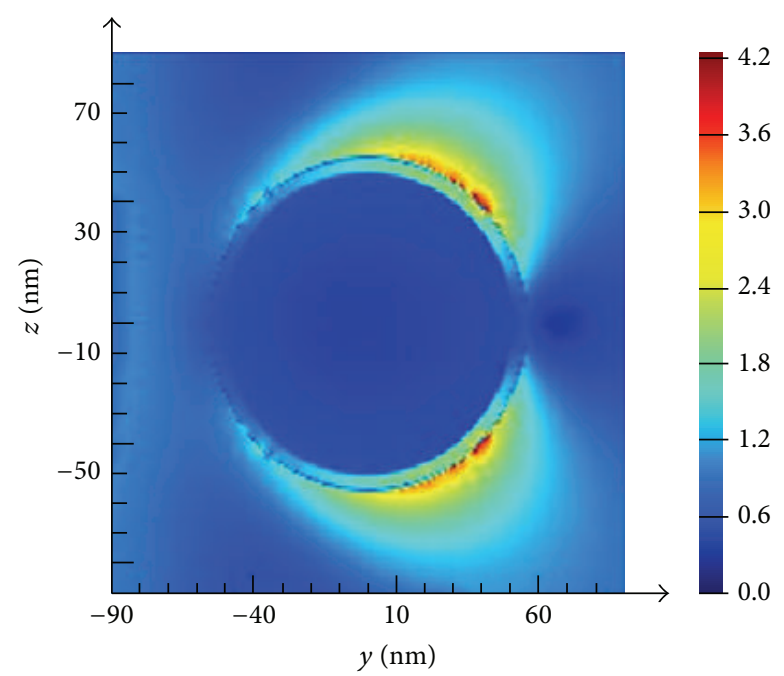

(c)

Figure 4: Snapshot of $E$ field near the gold-silver core-shell: (a) Au NS of radius $50 \mathrm{~nm}$ at $535 \mathrm{~nm}$, (b) Au NS of radius $50 \mathrm{~nm}$ with Ag shell of thickness $5 \mathrm{~nm}$ at $535 \mathrm{~nm}$, and (c) Au NS of radius $50 \mathrm{~nm}$ with Ag shell of thickness $5 \mathrm{~nm}$ at $435 \mathrm{~nm}$.

where $c$ is the speed of light. While our simulation computes both absorption and scattering, they are related via the Kramers-Kronig equation [32].

\section{Simulation Results}

FDTD simulations allow us to assess design concept and reduce reliance upon costly experimental prototyping. First, we investigate the absorption spectra of the building blocks for more complex nanostructures with broad SPR absorption.

\subsection{SPR Absorption Spectra of Silver Nanowire. Figure 1} shows the SPR absorption spectrum of silver nanowires, or nanorods of infinite length, with varying radii over the $300 \mathrm{~nm}$ to $400 \mathrm{~nm}$ range. The absorption spectrum is calculated and expressed in terms of absorption cross section as a function of light wavelength. An Ag nanowire of radius $15 \mathrm{~nm}$ has peak absorption at $340 \mathrm{~nm}$, with an absorption cross section of approximately $60 \mathrm{~nm}$. Upon increasing the radius of the nanowire to $25 \mathrm{~nm}, 35 \mathrm{~nm}$, and $45 \mathrm{~nm}$, the maximum absorption cross section grew as well to approximately $110 \mathrm{~nm}, 120 \mathrm{~nm}$, and $150 \mathrm{~nm}$, respectively. However, the absorption peak position as well as full width at half maximum (FWHM) of the SPR band did not change significantly, so all Ag nanowires have strong absorption in the ultraviolet region.

Furthermore, comparison of the SPR absorption of an Ag nanowire with the absorption spectrum calculated by Mie theory demonstrated consistency between the simulation results and theory [29].

3.2. SPR Absorption Spectra of Gold Nanoparticles. Figure 2 shows the SPR absorption spectrum of gold nanoparticles with varying radii over the $450 \mathrm{~nm}$ to $700 \mathrm{~nm}$ range. Au NPs 


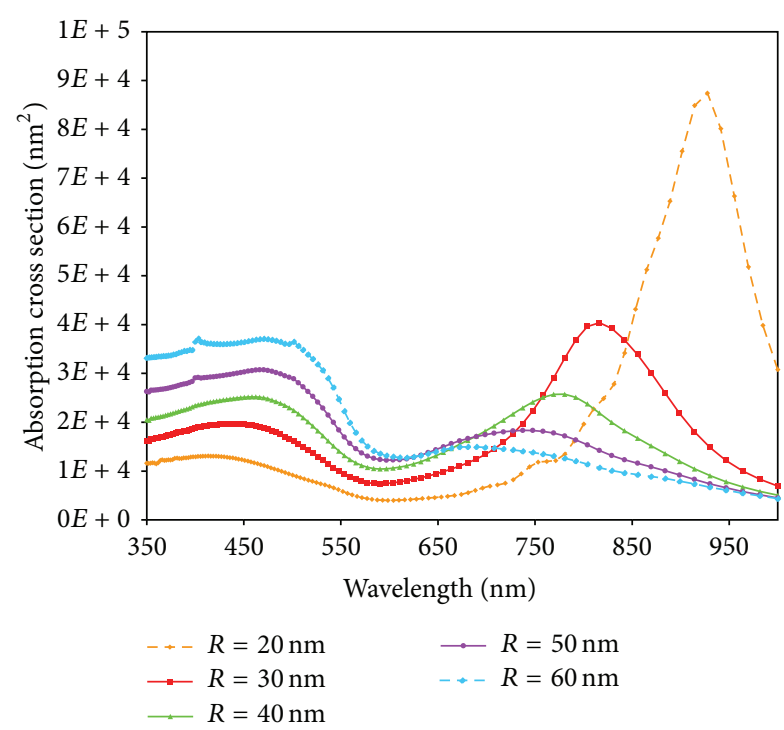

FIGURE 5: SPR absorption of gold NRs as a function of radius (constant length: $200 \mathrm{~nm}$ ).

of radius $30 \mathrm{~nm}, 40 \mathrm{~nm}, 50 \mathrm{~nm}$, and $60 \mathrm{~nm}$ resulted in peak absorption cross sections of approximately $5.0 \times 10^{3} \mathrm{~nm}^{2}$, $1.0 \times 10^{4} \mathrm{~nm}^{2}, 2.2 \times 10^{4} \mathrm{~nm}^{2}$, and $3.2 \times 10^{4} \mathrm{~nm}^{2}$, respectively, at around $525 \mathrm{~nm}$. As with $\mathrm{Ag}$ nanowires, changing the size of the Au NPs does not change the SPR peak position noticeably; thus, all Au NPs have strong absorption in the visible region. The absorption spectra have been shown to match experimental data [21].

3.3. Expanded SPR Absorption of Gold Nanoparticles with Silver Shell. In Figure 3, we compare the SPR absorption spectra of an $\mathrm{Au}$ NS of radius $50 \mathrm{~nm}$ and that of an $\mathrm{Au} / \mathrm{Ag}$ core/shell $(50 \mathrm{~nm}$ core radius and $3 \mathrm{~nm}$ and $5 \mathrm{~nm}$ shell thickness) in the wavelength range of $300 \mathrm{~nm}$ to $700 \mathrm{~nm}$. The addition of the $3 \mathrm{~nm}$ Ag shell causes an increase in absorption cross section in the UV region of the spectrum by $\sim 3 \times 10^{3} \mathrm{~nm}^{2}$, and increasing the shell thickness further to $5 \mathrm{~nm}$ increases the absorption by an additional $\sim 2 \times 10^{3} \mathrm{~nm}^{2}$. Meanwhile, the SPR peak due to Au remains around $525 \mathrm{~nm}$. The Ag shell does not affect the absorption in the visible region that is dominated by $\mathrm{Au}$, as expected.

Figure 4 shows snapshots of the $E$ field near the goldsilver core-shell for Au NSs of radius $50 \mathrm{~nm}$ : (a) with no shell at $535 \mathrm{~nm}$, (b) with Ag shell of thickness $5 \mathrm{~nm}$ at $535 \mathrm{~nm}$, and (c) with Ag shell of thickness $5 \mathrm{~nm}$ at $435 \mathrm{~nm}$. Figure 4(c) clearly demonstrates the enhancement of $E$ field due to the silver shell.

3.4. Tunable SPR Absorption of Gold Nanorods. Figure 5 shows the SPR absorption spectra of a series of Au NRs with varying aspect ratios, from 3.3 to 10 , in the wavelength region of $350 \mathrm{~nm}$ to $1000 \mathrm{~nm}$. Two major SPR peaks are observed, as expected-one is due to the longitudinal mode of oscillation (red-NIR) and another due to the transverse mode (UV-visible) [33]. For a fixed NR length of $200 \mathrm{~nm}$, as the radius of the NR increases from $20 \mathrm{~nm}$ to $50 \mathrm{~nm}$, the SPR absorption cross section decreases in the red and NIR regions but increases in the UV-visible region. The calculated spectra are consistent with experimental results [21].

3.5. Application of Gold Nanoparticles and Nanorods for Photoelectrochemical (PEC) Water Splitting. Collecting and storing solar energy in the form of chemical energy are highly desirable because it is effective for both conversion and storage, two of the major challenges in solar energy technology. This can be accomplished through techniques such as PEC water splitting, for which $\mathrm{TiO}_{2}$ has commonly been used as the photoanode due to its stability and low cost. However, its large bandgap greatly limits light absorption in the visible region. Visible light absorption can be potentially enhanced via the application of metal nanostructures, which has been demonstrated experimentally [6]. The mechanisms behind this enhancement are still not well understood. To help understand the effect of metal nanostructures on the PEC performance of metal oxides such as $\mathrm{TiO}_{2}$, we simulated the SPR of $\mathrm{Au}$ NPs and NRs on a $\mathrm{TiO}_{2}$ substrate and correlated it with experimental results of NS-sensitized $\mathrm{TiO}_{2}$.

Figure 6 illustrates the SPR absorption spectra and $E$ field distribution of $\mathrm{Au} \mathrm{NP}$ and NR attached to a $\mathrm{TiO}_{2}$ substrate, in a water medium, and shows comparison of the absorption spectra of bare $\mathrm{TiO}_{2}$ substrate with that of $\mathrm{TiO}_{2}$ substrate decorated with Au NP and NR. The enhancement of solar light absorption due to Au NPs and NRs is observed over the entire visible light region and can be up to a few thousand folds. Figure 6(b) shows $E$ field density plot on the $y-z$ plane for (left) Au NP ( $R=20 \mathrm{~nm})$ at $535 \mathrm{~nm}$ and (right) Au NR $(R=8 \mathrm{~nm}$ and $L=40 \mathrm{~nm})$ at $750 \mathrm{~nm}$. The incident light propagates in the $y$ direction (horizontal). The field is the strongest at the interface of $\mathrm{Au}$ and $\mathrm{TiO}_{2}$, which indicates that the enhancement in visible light absorption may be due to strong interaction between $\mathrm{Au}$ and $\mathrm{TiO}_{2}$. This either results in electron injection from $\mathrm{Au}$ to $\mathrm{TiO}_{2}$ or increases the light absorption of $\mathrm{TiO}_{2}$ itself due to bandgap states.

3.6. Application of Silver Nanoparticles in Si Thin-Film Solar Cells. Silver nanoparticle arrays have been applied for the enhancement of electric fields near the surface of thinfilm silicon solar cells (local field enhancement), leading to increased efficiency [10]. In this study, we aimed to investigate the effects of a number of key factors on the SPR of Ag NP arrays to optimize their local field enhancement.

The quantum efficiency of a solar cell, $\mathrm{QE}(\lambda)$, is defined as

$$
\mathrm{QE}(\lambda)=\frac{P_{\mathrm{abs}}(\lambda)}{P_{\mathrm{in}}(\lambda)},
$$

where $P_{\text {in }}(\lambda)$ and $P_{\text {abs }}(\lambda)$ are the power of the incident light and absorbed light within the Si solar cell, respectively, at a wavelength $\lambda$. The integrated quantum efficiency, IQE, is defined as

$$
\mathrm{IQE}=\frac{\int(\lambda / h c) \mathrm{QE}(\lambda) I_{\mathrm{AM} 1.5}(\lambda) d \lambda}{\int(\lambda / h c) I_{\mathrm{AM} 1.5}(\lambda) d \lambda}
$$




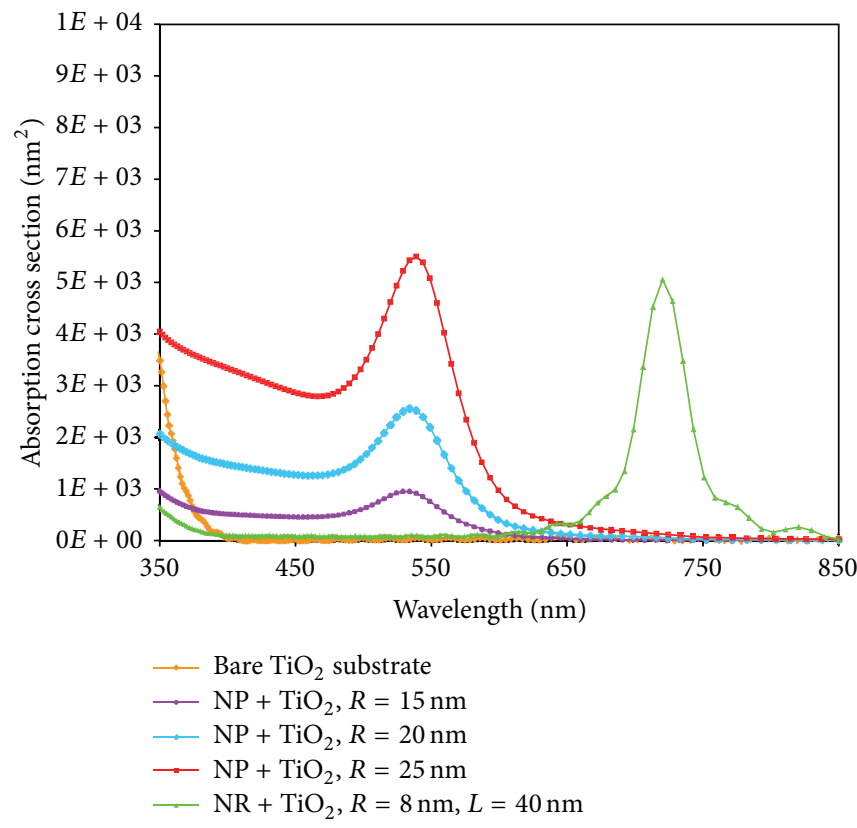

(a)
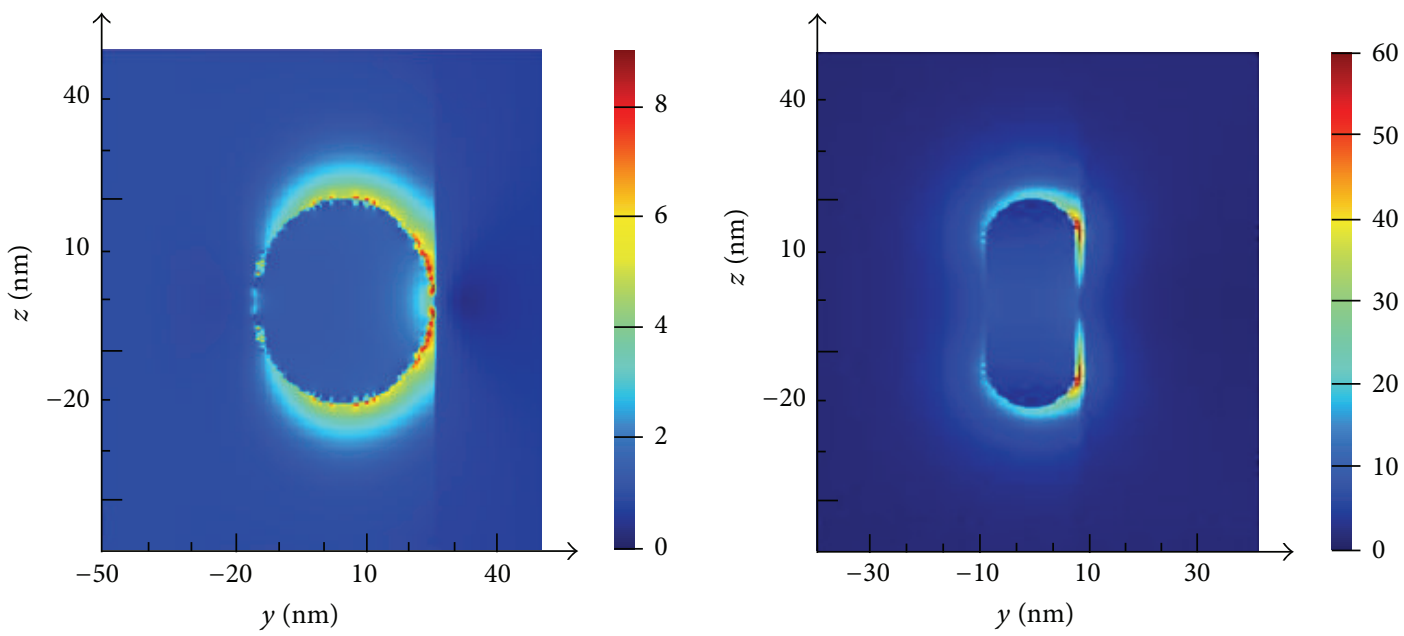

(b)

Figure 6: Absorption and $E$ field enhancement due to $\mathrm{Au} \mathrm{NP}$ and $\mathrm{NR}$, all in water (a). Comparison of absorption spectra of bare $\mathrm{TiO}_{2}$ substrate with that of $\mathrm{TiO}_{2}$ substrate plus Au NP NS and NR. (b) $E$ field density plot on the $y$ - $z$ plane for (left) Au NP $(R=20 \mathrm{~nm})$ at $549 \mathrm{~nm}$ and (right) Au NR ( $R=8 \mathrm{~nm}$ and $L=40 \mathrm{~nm})$ at $728 \mathrm{~nm}$.

where $h$ is Plank's constant, $c$ is the speed of light in free space, and $I_{\mathrm{AM} 1.5}$ is the air mass 1.5 soar spectrum. To compare the efficiency of solar cell with metal particles with that of a bare solar cell, we define QE enhancement $g(\lambda)$ and IQE enhancement $G$ as follows:

$$
\begin{aligned}
g(\lambda) & =\frac{\mathrm{QE}_{\text {nanoparticle }}(\lambda)}{\mathrm{QE}_{\text {bare } \mathrm{Si}}(\lambda)}, \\
G & =\frac{\mathrm{IQE}_{\text {nanoparticle }}}{\mathrm{IQE}_{\text {bare } \mathrm{Si}}} .
\end{aligned}
$$

The absorption per unit volume can be calculated from the divergence of the Poynting vector:

$$
L=-0.5 \omega|E|^{2} \operatorname{imag}(\varepsilon) .
$$

We first studied the enhancement of integrated quantum efficiency, $G$, as a function of Ag NP radius and center-tocenter spacing, or period, in an array to find the optimal parameters. Figure 7 shows the enhancement of IQE due to $\mathrm{Ag} \mathrm{NP}$ arrays with radii of $37.5 \mathrm{~nm}, 75 \mathrm{~nm}$, and $150 \mathrm{~nm}$ and periods ranging between 100 and $800 \mathrm{~nm}$. The large enhancements occur with periods at $100 \mathrm{~nm}, 350 \mathrm{~nm}$, and $600 \mathrm{~nm}$, respectively. Furthermore, NPs with radius $75 \mathrm{~nm}$ can be seen to have the most significant overall enhancement.

A more in-depth investigation into the effect of array period is shown in Figure 8, which compares the enhancement of Ag NP arrays with radius $75 \mathrm{~nm}$ and period between NPs of $225,325,425$, and $525 \mathrm{~nm}$, to the solar spectrum. It can be seen that a period of $325 \mathrm{~nm}$, which is consistent with the estimate as shown in Figure 7, has a scattering spectrum 


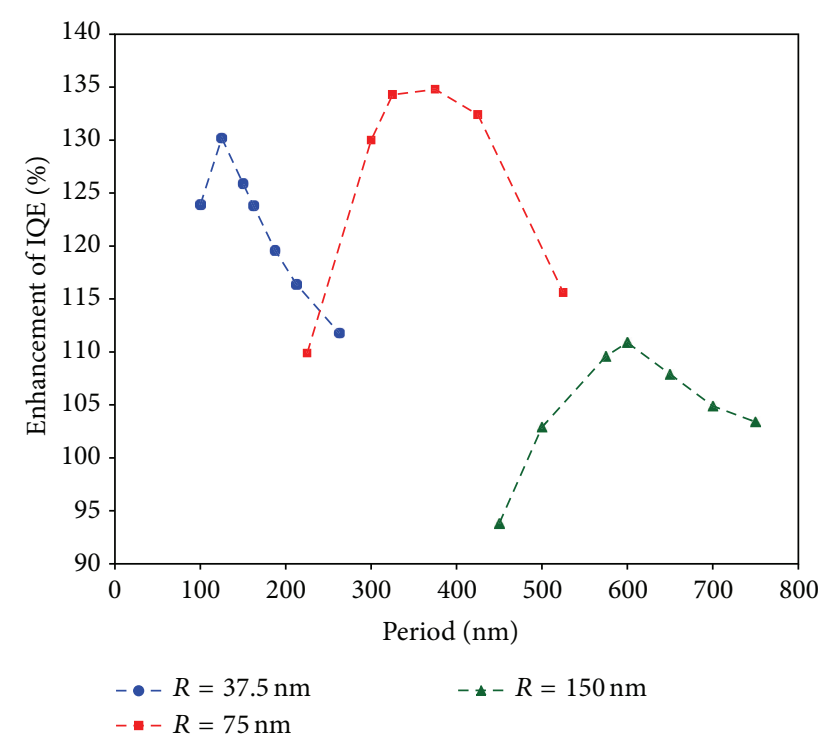

FIGURE 7: Enhancement of IQE as a function of Ag NP radius and period.

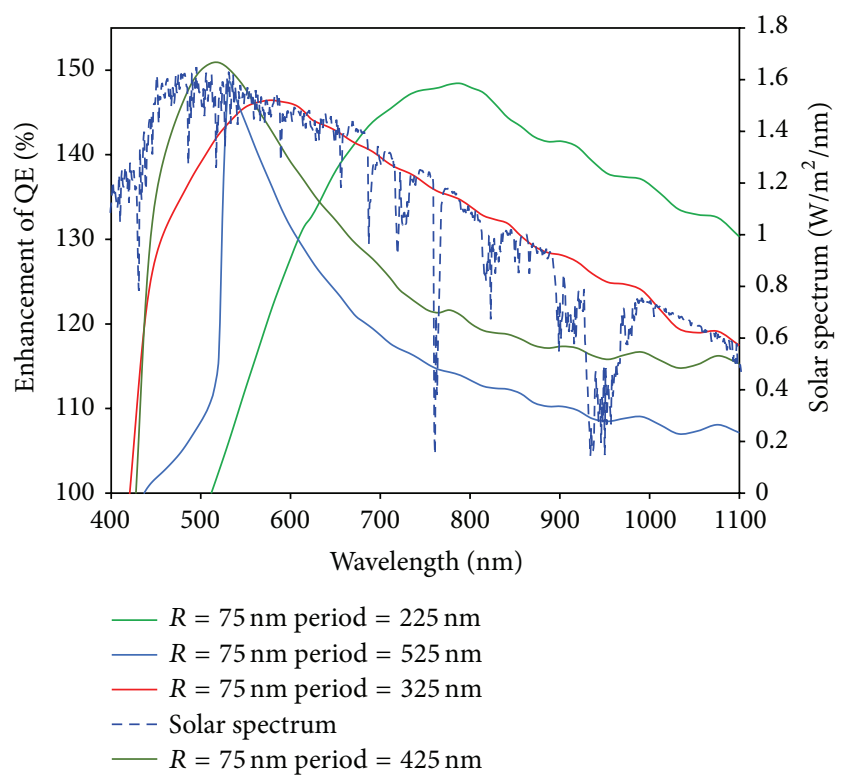

FIGURE 8: Enhancement of Si thin-film solar cells efficiency over the $400 \mathrm{~nm}$ to $1100 \mathrm{~nm}$ range as function of NP array period from $225 \mathrm{~nm}$ to $525 \mathrm{~nm}$.

most closely matching the solar spectrum and is thusly the most advantageous spacing for Si solar cell enhancement.

Figures 9(a) and 9(b) show top and side views of an Ag NP array on a Si thin-film solar cell. Figures 9(c) and 9(d) illustrate the power absorption per volume by the Si substrate, with and without an Ag NP. There is clear and significant enhancement of light absorption that is caused by the Ag NP array.

3.7. Broad SPR Absorption of Complex Assembled Structure to Match Solar Spectrum. After simulating the SPR spectra of a large number of simple and complex metal nanostructures, we reached the key conclusion that a complex metal structure is necessary for broad SPR absorption. A number of design ideas were evaluated using computer simulation to avoid costly experimental iteration in the lab. Figure 10 shows the final complex gold nanostructure which was built using $\mathrm{Au}$ NRs and NPs. The shape and size of each NP and NR were adjusted until the absorption spectrum closely matched the solar spectrum (300-1500 nm) as shown in Figure 11. The assembled nanostructure has a large Au NP core with radius of 55 and a silica shell of $15 \mathrm{~nm}$, which reduces the interaction between the Au core and the Au NRs and smaller Au NPs. The six small Au NPs with radius of $30 \mathrm{~nm}$ were attached to the top, bottom, left, right, front, and back of the large $\mathrm{Au}-\mathrm{SiO}_{2}$ core-shell. In addition, eight Au NRs with length to radius ratio ranging from 2 to 10 were attached to the core-shell. The dimensions of the $\mathrm{Au}$ NRs are given in the table as an inset of Figure 10. A more detailed discussion of the rationale and design principle will be given in Section 4 later.

Comparison of the spectrum of the Au core itself and that of the assembled nanostructure (Figure 11) clearly demonstrates that one can significantly broaden the absorption spectrum by combining a number of simple nano-building blocks such as NSs and NRs. The $\mathrm{SiO}_{2}$ insulation shell, used to reduce the interaction between the core and other components of the assembled nanostructure, enables us to tune the spectrum relatively independently, which is a critical original idea developed in this project.

\section{Discussion}

4.1. Design Strategy for Metal Nanostructures with Broad SPR Absorption. As mentioned in Section 3, one of our key findings is that a complex metal structure is necessary for broad SPR absorption. This is due to the fact that the SPR is very sensitive to the shape (or symmetry) and, to a lesser degree, the size of metal nanostructures [34]. This inspired the idea of using NPs of different shape and size to achieve a combined spectrum that meets our requirements.

While in principle many complex structures may produce a broad SPR spectrum, one must take into account the feasibility of fabrication. In our design, we chose to use two very common and easily synthesizable gold nanostructures, NPs and NRs, as the basic building blocks for the complex assembled structure. Our plan was to use a large core gold NP to provide strong SPR in the green region (around $525 \mathrm{~nm}$ ) and use NRs of various aspect ratios to afford strong red and NIR absorption. However, when we simply assembled $\mathrm{Au}$ NSs and NRs, the resulting spectrum was not as expected. One critical factor in the complex structure is the interaction between its components, which must be carefully controlled. The absorption spectrum of the assembled structure with strong interaction between the NSs and NRs is not a simple sum of the spectra of its components. Rather, the spectrum is distorted in an undesired way due to the interaction [35]. With this in mind, we used a silica $\left(\mathrm{SiO}_{2}\right)$ layer to fine-tune interaction between the individual components to produce a more desired spectrum of the complex structure. The final structure that has the SPR spectrum best matching the solar 


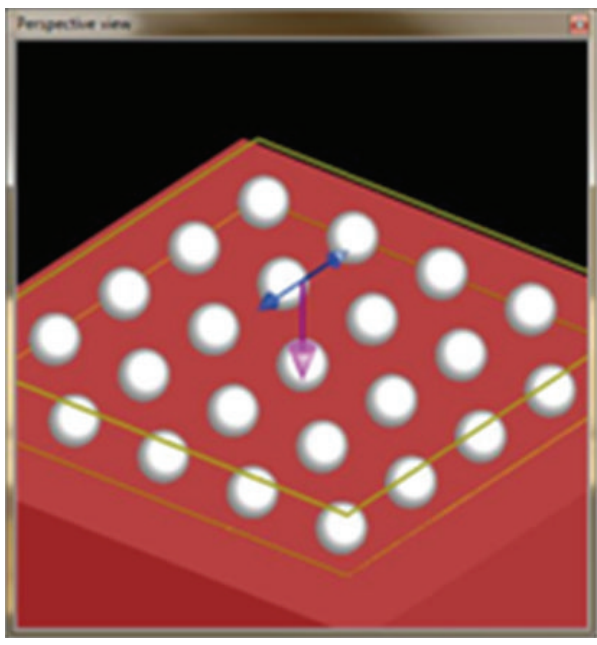

(a)

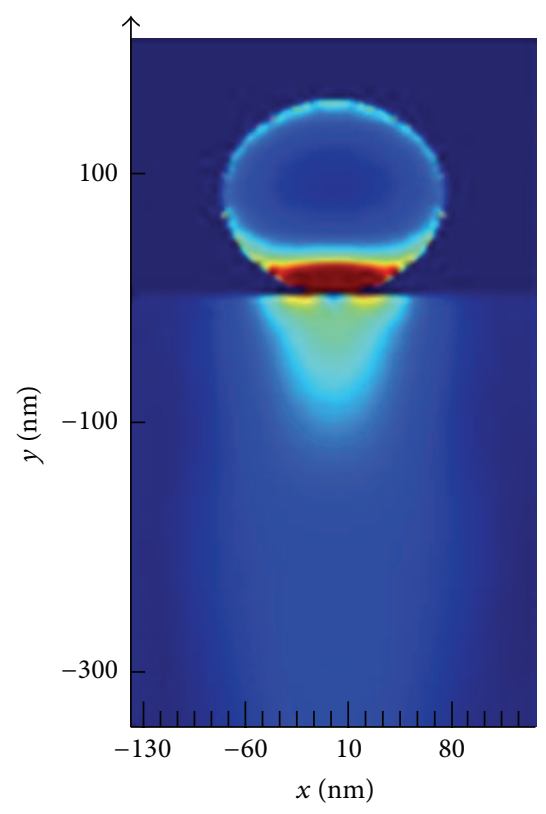

(c)

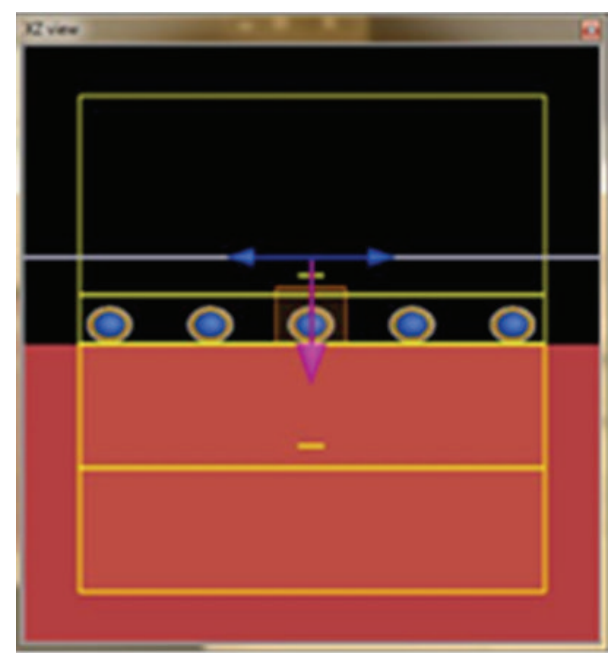

(b)

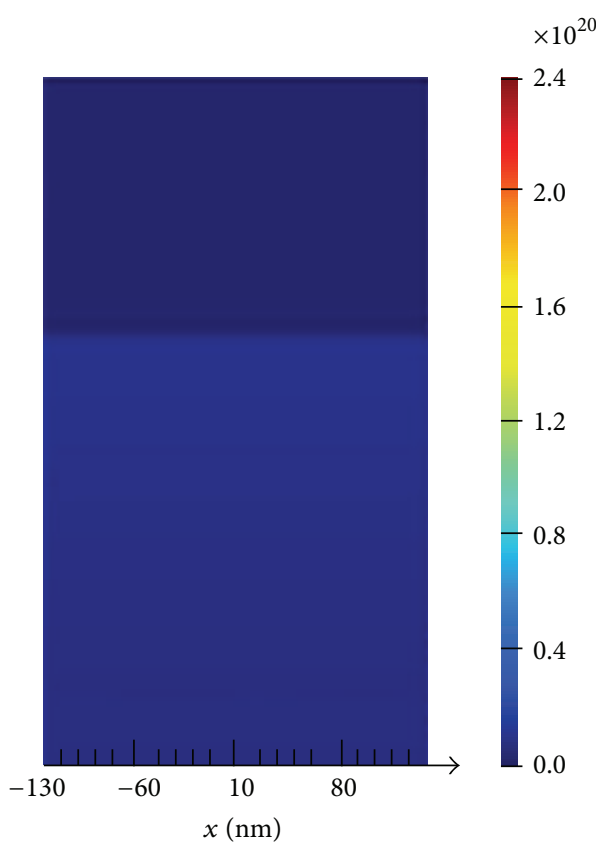

(d)

Figure 9: Si thin-film solar cell with array of Ag NPs: (a) top view, (b) side view, and (c) and power absorption per volume with (c) and without (d) Ag NPs.

spectrum is shown in Figure 10, with its SPR spectrum shown in Figure 11. The success of the design is thus based on both controlling the interaction between the individual components and tuning the shape and size of its components to control the SPR of the final assembled nanostructure. We used only simple building blocks to construct the complex structure in order to enable laboratory fabrication and ultimately mass production.

4.2. Insight into the Broadened SPR for Solar Energy Conversion. Experimental data on the optical properties of metal nanostructures demonstrated that the basic idea of assembling complex MNs from simple building blocks truly works, and it provided new insight into the design strategy for such MNs. It should be noted that MNs such as NPs and NRs are not simply bare or naked particles and they usually have species on their surfaces for stabilization. These species impact the interaction between the components and thus introduce additional challenges in the design of complex structures.

4.3. Silver Nanoparticle Arrays for Optimal Local Field Scattering Enhancement. Enhancing the efficiency of Si thin-film solar cells requires that the scattering spectra of MNs match the solar spectrum. Sweeping the radius and period of the Ag NP in an array, we were able to find the combination 


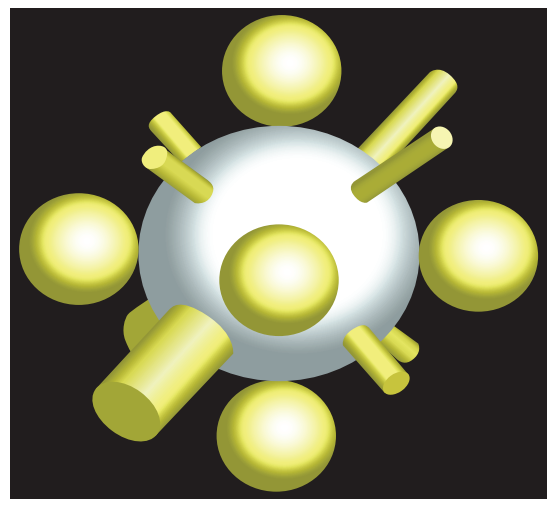

\begin{tabular}{lcc}
\hline $\begin{array}{l}\text { Radius } \\
(\mathrm{nm})\end{array}$ & $\begin{array}{c}\text { Length } \\
(\mathrm{nm})\end{array}$ & $L / R$ \\
\hline 20 & 40 & 2 \\
20 & 70 & 3.5 \\
8 & 40 & 5 \\
7 & 42 & 6 \\
7 & 49 & 7 \\
6.5 & 52 & 8 \\
8.4 & 80 & 9.5 \\
6.5 & 78 & 12 \\
\hline \multicolumn{3}{c}{ Table: Au NR dimensions }
\end{tabular}

FIGURE 10: Assembled Au nanostructure whose SPR absorption closely matches the solar spectrum. The table specifies the dimensions of the NRs.

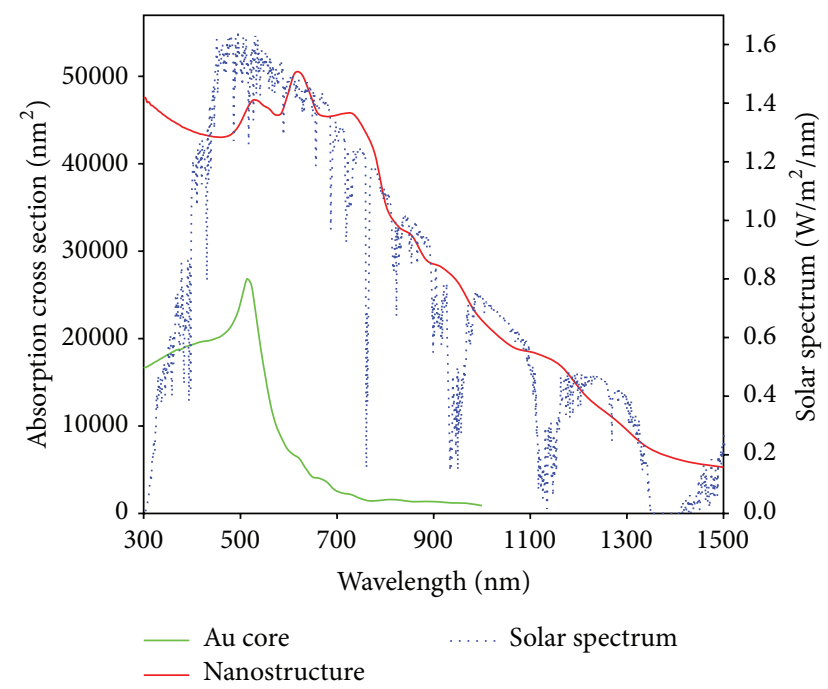

FIGURE 11: SPR absorption spectrum of the assembled nanostructure closely matching the solar spectrum in the visible and NIR regions, from $300 \mathrm{~nm}$ to $1500 \mathrm{~nm}$.

of parameters that optimize the forward scattering of light by Ag NP into the silicon. After first investigating the array IQE as a function of NP size, it was determined that a radius of $75 \mathrm{~nm}$ yielded optimal enhancement (Figure 7). We then studied the effects of the array's period on its scattering spectrum. It was found that a period of $325 \mathrm{~nm}$ yields the closest scattering spectrum to the solar spectrum (Figure 8), and thus the highest quantum efficiency enhancement of a thin-film silicon solar cell.

\section{Conclusion}

In summary, we have applied advanced simulation techniques to study the fundamental SPR properties of plasmonic $\mathrm{MN}$ and used the knowledge gained to design new complex nanostructures with unique absorption and scattering properties. In particular, a novel structure consisting of a large Au NP core and NRs plus smaller NPs with a silica spacing layer has been successfully developed. The SPR absorption of the complex nanostructures closely matches the solar spectrum from $300 \mathrm{~nm}$ to $1500 \mathrm{~nm}$, a region containing $90 \%$ of solar energy [36]. This structure is highly promising for solar energy conversion applications.

We also simulated the application of Ag NP arrays for the enhancement of thin-film silicon solar cells to study the effect of variables such as size and period on their scattering spectra. It was found that Ag NPs of radius $75 \mathrm{~nm}$ and period $325 \mathrm{~nm}$ result in a scattering spectrum that most closely matches the solar spectrum, leading to an up to $35 \%$ enhancement of the integrated quantum efficiency of the solar cells. In addition, it was shown that the cores of Ag NPs can be replaced with cheaper materials or removed entirely with minimal decrease in efficiency, enabling reduced-cost enhancement of solar cells. Our work demonstrates the potential of MNs to enhance both MO and thin-film silicon solar cells for efficient, broadband, and reduced-cost solar energy conversion.

\section{Conflict of Interests}

The authors declare that there is no conflict of interests regarding the publication of this paper.

\section{Acknowledgments}

Kristine A. Zhang is grateful to Mr. Karl Woll and Dr. Tom Yuvinsky for their help. Yat Li thanks the US NSF for financial support.

\section{References}

[1] Q. Schiermeier, J. Tollefson, T. Scully, A. Witze, and O. Morton, "Energy alternatives: electricity without carbon," Nature, vol. 454, no. 7206, pp. 816-823, 2008.

[2] B. O'Regan and M. Graetzel, "Low-cost, high-efficiency solar cell based on dye-sensitized colloidal $\mathrm{TiO}_{2}$ films," Nature, vol. 353, no. 6346, pp. 737-740, 1991.

[3] M. A. Green, Third Generation Photovoltaics: Advanced Solar Energy Conversion, Springer, New York, NY, USA, 2003. 
[4] A. Shah, P. Torres, R. Tscharner, N. Wyrsch, and H. Keppner, "Photovoltaic technology: the case for thin-film solar cells," Science, vol. 285, no. 5428, pp. 692-698, 1999.

[5] F. E. Osterloh and B. A. Parkinson, "Recent developments in solar water-splitting photocatalysis," MRS Bulletin, vol. 36, no. 1, pp. 17-22, 2011.

[6] Y.-C. Pu, G. M. Wang, K.-D. Chang et al., "Au nanostructuredecorated $\mathrm{TiO}_{2}$ nanowires exhibiting photoactivity across entire UV-visible region for photoelectrochemical water splitting," Nano Letters, vol. 13, no. 8, pp. 3817-3823, 2013.

[7] P. Wang, S. M. Zakeeruddin, J. E. Moser, M. K. Nazeeruddin, T. Sekiguchi, and M. Grätzel, "A stable quasi-solid-state dyesensitized solar cell with an amphiphilic ruthenium sensitizer and polymer gel electrolyte," Nature Materials, vol. 2, no. 6, pp. 402-407, 2003.

[8] A. J. Nozik, "Quantum dot solar cells," Physica E: Lowdimensional Systems and Nanostructures, vol. 14, no. 1-2, pp. 115120, 2002.

[9] G. D. Barber, P. G. Hoertz, S.-H. A. Lee et al., "Utilization of direct and diffuse sunlight in a dye-sensitized solar cellsilicon photovoltaic hybrid concentrator system," The Journal of Physical Chemistry Letters, vol. 2, no. 6, pp. 581-585, 2011.

[10] C. Rockstuhl, S. Fahr, and F. Lederer, "Absorption enhancement in solar cells by localized plasmon polaritons," Journal of Applied Physics, vol. 104, no. 12, Article ID 123102, 2008.

[11] Y. Tian and T. Tatsuma, "Mechanisms and applications of plasmon-induced charge separation at $\mathrm{TiO}_{2}$ films loaded with gold nanoparticles," Journal of the American Chemical Society, vol. 127, no. 20, pp. 7632-7637, 2005.

[12] H. A. Atwater, S. Maier, A. Polman, J. A. Dionne, and L. Sweatlock, "The new 'p-n junction': plasmonics enables photonic access to the nanoworld," MRS Bulletin, vol. 30, pp. 385-389, 2005.

[13] A. Furube, L. Du, K. Hara, R. Katoh, and M. Tachiya, "Ultrafast plasmon-induced electron transfer from gold nanodots into $\mathrm{TiO}_{2}$ nanoparticles," Journal of the American Chemical Society, vol. 129, no. 48, pp. 14852-14853, 2007.

[14] J. M. Chen, Y. P. Zou, Y. F. Li et al., "Improving the photoelectrochemical performance of polythiophene sensitized $\mathrm{TiO}_{2}$ electrode by modification with gold nanoparticles," Chemical Physics Letters, vol. 460, no. 1-3, pp. 168-172, 2008.

[15] H. A. Atwater and A. Polman, "Plasmonics for improved photovoltaic devices," Nature Materials, vol. 9, no. 3, pp. 205213, 2010.

[16] Z. H. Chen, Y. B. Tang, C. P. Liu et al., "Vertically aligned ZnO nanorod arrays sentisized with gold nanoparticles for schottky barrier photovoltaic cells," Journal of Physical Chemistry C, vol. 113, no. 30, pp. 13433-13437, 2009.

[17] H. Choi, W. T. Chen, and P. V. Kamat, "Know thy nano neighbor. Plasmonic versus electron charging effects of metal nanoparticles in dye-sensitized solar cells," ACS Nano, vol. 6, no. 5, pp. 4418-4427, 2012.

[18] J.-C. Tinguely, I. Sow, C. Leiner et al., “Gold Nanoparticles for plasmonic biosensing: the role of metal crystallinity and nanoscale roughness," BioNanoScience, vol. 1, no. 4, pp. 128-135, 2011.

[19] R. Gaughan, "Metal nanoparticles offer new imaging method," Photonics Spectra, vol. 36, p. 93, 2002.

[20] D. Derkacs, S. H. Lim, P. Matheu, W. Mar, and E. T. Yu, "Improved performance of amorphous silicon solar cells via scattering from surface plasmon polaritons in nearby metallic nanoparticles," Applied Physics Letters, vol. 89, no. 9, Article ID 093103, 2006.

[21] P. K. Jain, K. S. Lee, I. H. El-Sayed, and M. A. El-Sayed, "Calculated absorption and scattering properties of gold nanoparticles of different size, shape, and composition: applications in biological imaging and biomedicine," Journal of Physical Chemistry B, vol. 110, no. 14, pp. 7238-7248, 2006.

[22] G. Mie, "Beiträge zur Optik trüber Medien, speziell kolloidaler Metallösungen," Annalen der Physik, vol. 330, no. 3, pp. 377-445, 1908.

[23] S. A. Maier, P. G. Kik, and H. A. Atwater, "Optical pulse propagation in metal nanoparticle chain waveguides," Physical Review $B$, vol. 67, no. 20, Article ID 205402, 2003.

[24] A. Mejdoubi, M. Malki, M. Essone Mezeme, Z. Sekkat, M. Bousmina, and C. Brosseau, "Optical scattering and electric field enhancement from core-shell plasmonic nanostructures," Journal of Applied Physics, vol. 110, no. 10, Article ID 103105, 2011.

[25] V. Myroshnychenko, J. Rodríguez-Fernández, I. PastorizaSantos et al., "Modelling the optical response of gold nanoparticles," Chemical Society Reviews, vol. 37, no. 9, pp. 1792-1805, 2008.

[26] A. Atkinson, J. McMahon, and G. Schatz, "FDTD studies of metallic nanoparticle systems," in Self-Organization of Molecular Systems, NATO Science for Peace and Security Series A: Chemistry and Biology, pp. 11-32, Springer, Amsterdam, The Netherlands, 2009.

[27] K. S. Yee, "Numerical solution of initial boundary value problems involving maxwell's equations in isotropic media," IEEE Transactions on Antennas and Propagation, vol. 14, no. 3, pp. 302-307, 1966.

[28] A. Taflove, A. Oskooi, and S. G. Hagness, Advances in FDTD Computational Electrodynamics: Photonics and Nanotechnology, Artech House, Norwood, Mass, USA, 2013.

[29] Lumerical, 2013, http://docs.lumerical.com/en/fdtd/nanowire resonance_tutorial.html?zoom_highlightsub=cross\%2Bsection.

[30] A. Taflove and S. G. Hagness, Computational Electrodynamics: The Finite-Difference Time-Domain Method, Artech House, Norwood, NJ, USA, 3rd edition, 2005.

[31] D. Sullivan, Electromagnetic Simulation Using the FDTD Method, IEEE Press, Piscataway, NJ, USA, 2013.

[32] V. Lucarini, J. Saarinen, K. Peiponen, and E. M. Vartiainen, Kramers-Kronig Relations in Optical Materials Research, Springer, New York, NY, USA, 2005.

[33] Y.-Y. Yu, S.-S. Chang, C.-L. Lee, and C. R. C. Wang, "Gold nanorods: electrochemical synthesis and optical properties," The Journal of Physical Chemistry B, vol. 101, no. 34, pp. 66616664, 1997.

[34] B. J. Wiley, S. H. Im, Z.-Y. Li, J. McLellan, A. Siekkinen, and Y. A. Xia, "Maneuvering the surface plasmon resonance of silver nanostructures through shape-controlled synthesis," Journal of Physical Chemistry B, vol. 110, no. 32, pp. 15666-15675, 2006.

[35] K. L. Kelly, A. A. Lazarides, and G. C. Schatz, "Computational electromagnetics of metal nanoparticles and their aggregates," Computing in Science \& Engineering, vol. 3, no. 4, pp. 67-73, 2001.

[36] A. Mecherikunnel and J. Richmond, "Spectral distribution of solar radiation,” NASA Technical Memorandum 82021, 1980. 

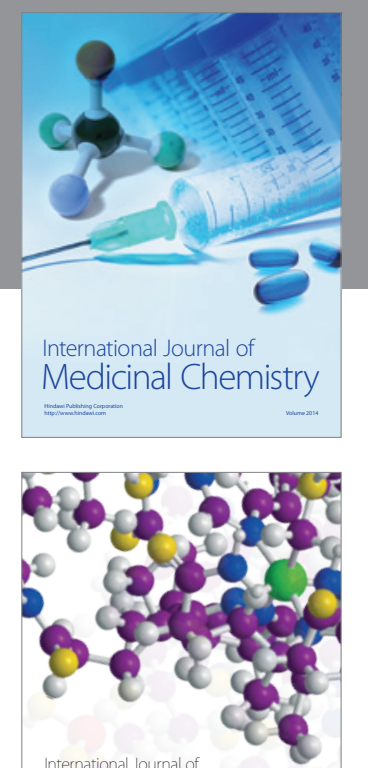

\section{Carbohydrate} Chemistry

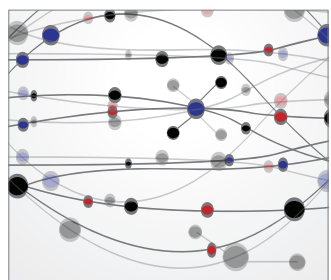

The Scientific World Journal
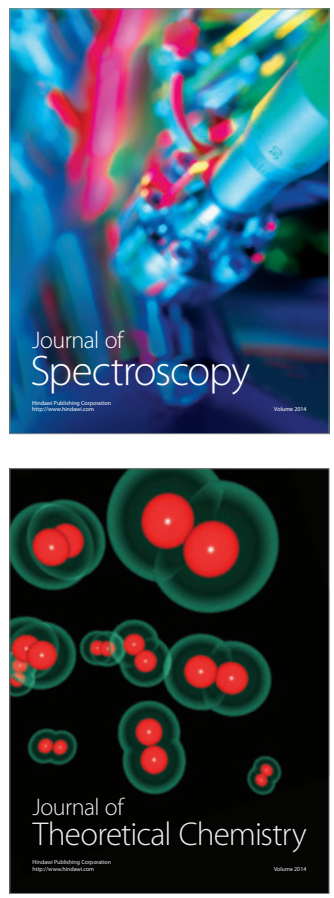
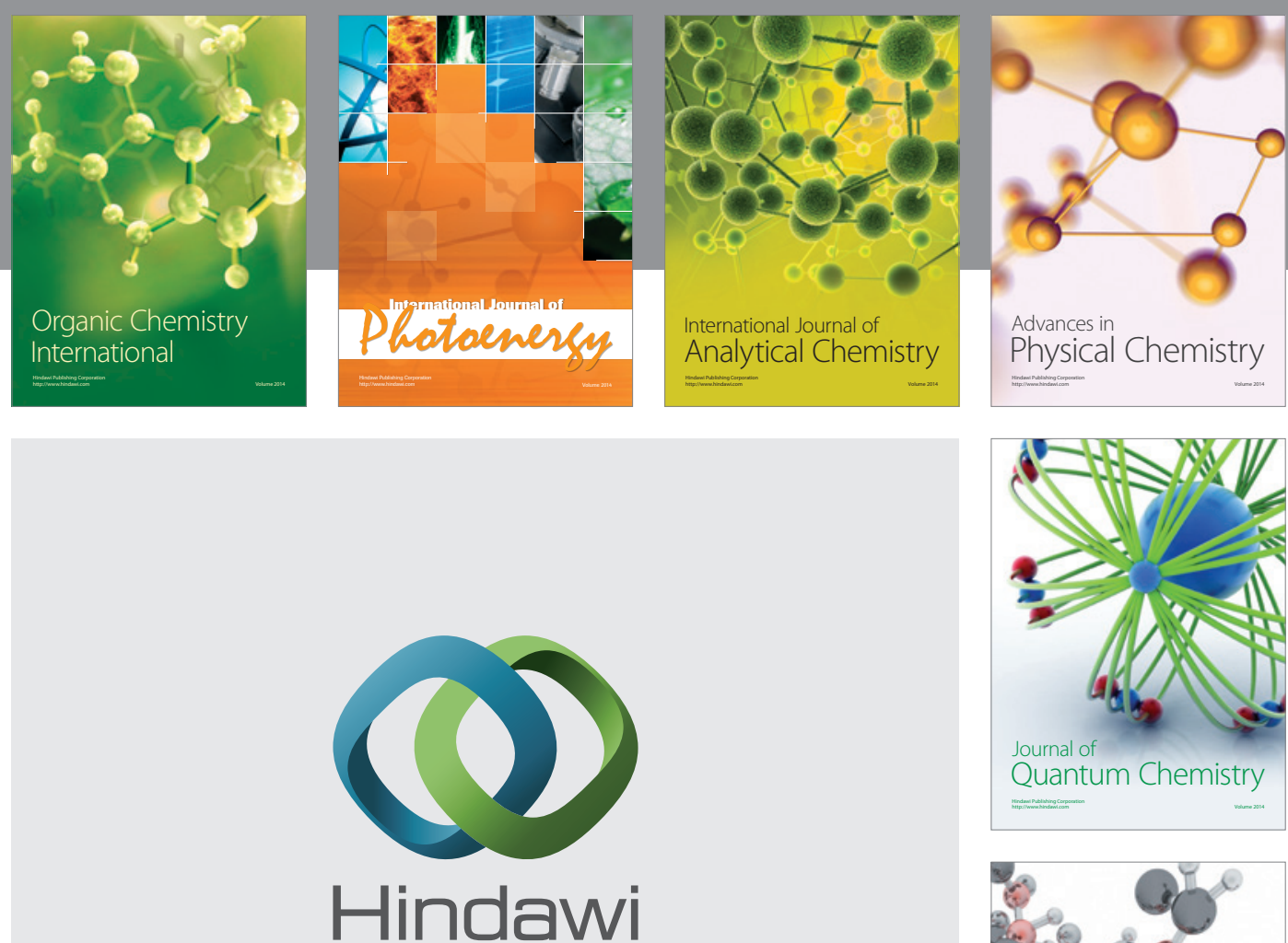

Submit your manuscripts at

http://www.hindawi.com

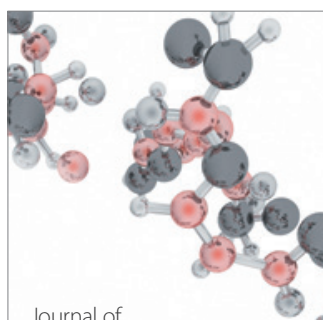

Analytical Methods

in Chemistry

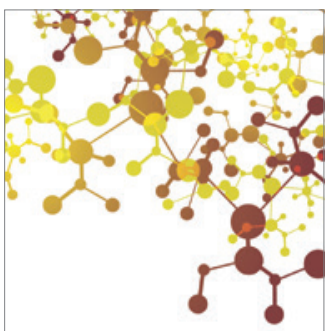

Journal of

Applied Chemistry

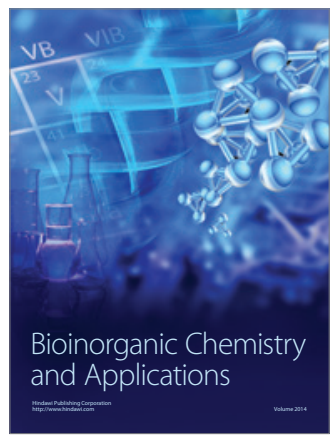

Inorganic Chemistry
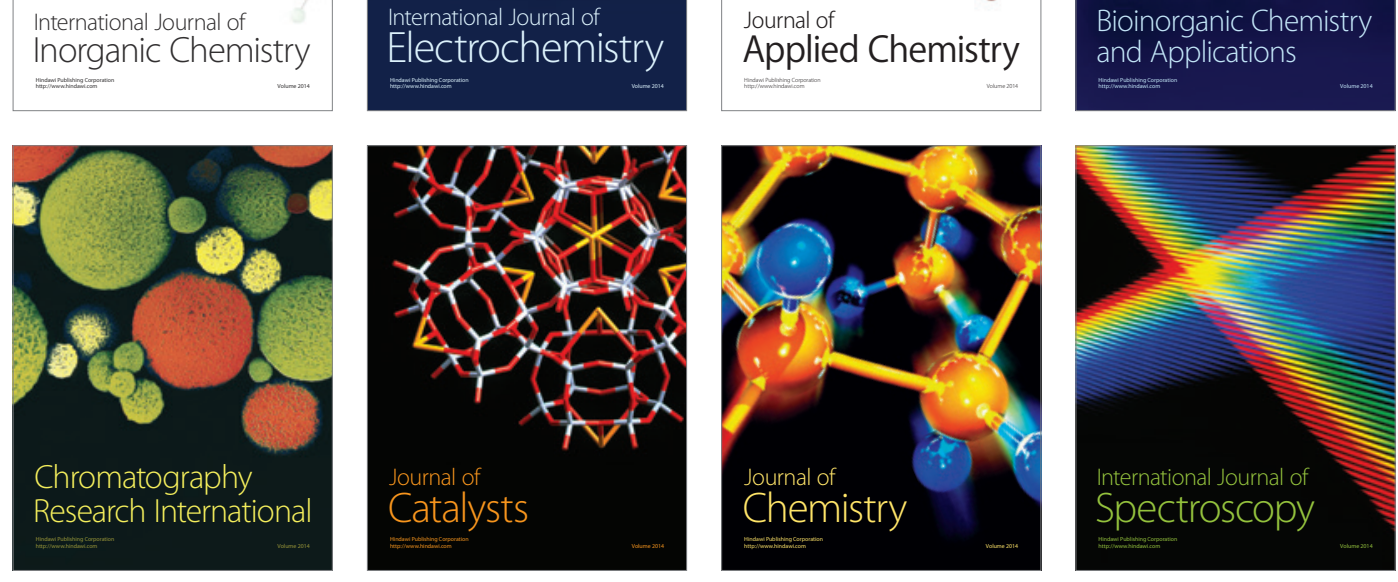